\title{
Targeting Innate Immunity to Combat Cutaneous Stress: The Vitiligo Perspective
}

\author{
Katia Boniface ${ }^{1 *}$, Thierry Passeron ${ }^{2,3}$, Julien Seneschal ${ }^{1,4}$ and Meri K. Tulic ${ }^{2}$ \\ 1 Univ. Bordeaux, INSERM, BMGIC, U1035, Immuno-dermatology Team, Bordeaux, France, 2 INSERM U1065, Centre \\ Méditerranéen de Médecine Moléculaire (C3M), Côte d'Azur University, Nice, France, ${ }^{3}$ Côte d'Azur University, Department of \\ Dermatology, CHU Nice, Nice, France, ${ }^{4}$ Department of Dermatology and Pediatric Dermatology, National Reference Center \\ for Rare Skin Disorders, Hôpital Saint-André, Bordeaux, France
}

\section{OPEN ACCESS}

Edited by:

J. Michelle Kahlenberg,

University of Michigan, United States

Reviewed by:

Jillian M. Richmond,

University of Massachusetts Medical School, United States

Rasheedunnisa Begum, Maharaja Sayajirao, University of Baroda, India

${ }^{*}$ Correspondence: Katia Boniface katia.boniface@u-bordeaux.fr

Specialty section: This article was submitted to Autoimmune and Autoinflammatory Disorders, a section of the journal

Frontiers in Immunology

Received: 01 October 2020 Accepted: 22 March 2021

Published: 14 April 2021

Citation:

Boniface K, Passeron T, Seneschal J and Tulic MK (2021) Targeting Innate Immunity to Combat Cutaneous

Stress: The Vitiligo Perspective.

Front. Immunol. 12:613056. doi: 10.3389/fimmu.2021.613056
Multiple factors are involved in the process leading to melanocyte loss in vitiligo including environmental triggers, genetic polymorphisms, metabolic alterations, and autoimmunity. This review aims to highlight current knowledge on how danger signals released by stressed epidermal cells in a predisposed patient can trigger the innate immune system and initiate a cascade of events leading to an autoreactive immune response, ultimately contributing to melanocyte disappearance in vitiligo. We will explore the genetic data available, the specific role of damage-associated-molecular patterns, and patternrecognition receptors, as well as the cellular players involved in the innate immune response. Finally, the relevance of therapeutic strategies targeting this pathway to improve this inflammatory and autoimmune condition is also discussed.

Keywords: innate immunity, vitiligo, PAMPs, DAMPs, ILC, DC, melanocytes

\section{INTRODUCTION}

Clinical, translational, and fundamental research studies performed over the last decade have tremendously improved our understanding of vitiligo physiopathology and new therapeutic perspectives are emerging for this disease which suffers from the lack of effective treatments. Vitiligo is a puzzling disease combining multiple intertwined components including environmental triggers, genetic predisposition, increased oxidative stress, and abnormal immune and inflammatory response $(1,2)$. Vitiligo is defined by the loss of epidermal melanocytes, nonetheless several cell subsets of immune and non-immune cells are involved to induce and/or contribute to their disappearance. Vitiligo skin is consistently associated with infiltration of T cells with a Th1/Tc1 skewed immune profile which target melanocytes $(3,4)$. Besides the role of the adaptive immune response, increasing data highlight a major role of innate immune cell subsets and their immunerelated pathways that could spark the induction of the disease in the "normal-appearing" skin. Therefore, this short review is focusing on the innate side of the disease, discussing how genetic and transcriptomic data revealed the importance of innate immunity in vitiligo, as well as the interplay between epidermal cells (keratinocytes and melanocytes) and innate immune cells to contribute to the initiation and/or progression of the disease through the release of danger signals, cytokines, and chemokines, leading to activation of the adaptive immune response and ultimately the loss of 
melanocyte. This better understanding now offers novel insight into the development of targeted therapies that could prevent the induction as well as the recurrence of the disease.

\section{GENETIC AND TRANSCRIPTOME DATA}

Genome wide association studies (GWAS) have identified over 50 susceptibility loci involved in melanogenesis and immunity in vitiligo patients (5). On the other hand, a delay in vitiligo age-ofonset over the past 30 years emphasizes the key role of environmental factors in triggering vitiligo in genetically predisposed individuals $(6,7)$. These GWAS studies not only demonstrated the implication of genes involved in melanogenesis and adaptive immunity but also revealed allelic variations in key genes involved in the innate immune responses, such as IFIH1, NLRP1, or TICAM1 (7-9).

Transcriptional analysis comparing gene expression profiles of skin from vitiligo patients with normal skin of healthy volunteers also emphasized the role of innate immunity (10, 11). Thus, natural killer (NK) cell activation markers, such as NKG2D, KLRC2, and KLRC4, ligands for NK receptor (CLEC2B), as well as markers of oxidative stress (CANP and POSTN) and innate immunity (DEFB103A) were shown to be increased in vitiligo skin (10). In our study, we also found a significant increase in NK receptors, including NKTR and KLRC1, as well as trends for increased EOMES (master regulator of NK cells), CCL20, and NK-related cytokines (TNF $\alpha$ and IL-15) (11). Interestingly, activation of these innate immunity markers was found in the non-lesional skin of vitiligo patients, suggesting that the activation of the innate immunity may be present throughout the entire skin surface of patients $(10,11)$.

Taken together, these data illustrate that vitiligo patients have genetic predisposition affecting their innate immune response in their apparent non-affected skin. Such findings may be indicative of a subclinical activation of innate immunity, loss of protective mechanisms to stress (such as defective unfolded protein response in target cells following endoplasmic reticulum stress), and/or increased sensitivity to endogenous or external stress, such as several damage-associated-molecular patterns (DAMPs) or pathogen-associated-molecular patterns (PAMPs) (12).

\section{ACTIVATION OF INNATE IMMUNE CELLS BY DANGER SIGNALS}

\section{DAMPs}

Several DAMPs have been detected in perilesional skin of vitiligo patients. Previous studies have shown that the chromatinassociated nuclear protein High-mobility group-box-1 (HMGB1) could be released by melanocytes under oxidative stress and could directly impact melanocyte survival (13-15). Additionally, HMGB1 could bind free DNA and HMGB1-DNA complexes and induce maturation of vitiligo patients' dendritic cells (DC), as well as the production of cytokines and chemokines by keratinocytes (16). Another candidate for sensing the immune system in vitiligo is calreticulin (CRT). In response to stress, CRT can localize at the surface of immune cells, affecting their antigen presentation, complement activation, and clearance of apoptotic cells. Moreover, CRT can translocate to the melanocyte surface when these cells undergo $\mathrm{H}_{2} \mathrm{O}_{2}$-mediated oxidative stress, increasing melanocyte immunogenicity. CRT may also enhance the immunogenic potential of melanocytes through their induction of pro-inflammatory cytokine production, such as IL6 and TNF $\alpha$ (17).

Heat shock proteins (HSP) are likely important candidates bridging stress to the skin with the innate immune response. Indeed, inducible HSP70 (HSP70i) released in the context of cellular stress, notably by epidermal cells (including keratinocytes and melanocytes) has been shown to accelerate the progression of the disease in a preclinical model (18-20). Likewise, modified HSP70i prevented or reversed vitiligo in a mouse and Sinclair Swine models of the disease $(21,22)$. In vitiligo patients, the expression of HSP70 in the skin correlated with disease activity and was lower in patients with stable disease (23). As discussed below, HSP70i could interact with several cell subsets, leading to their activation.

\section{Pattern Recognition Receptors}

PAMPs are critical in initiation of the innate immune response through activation of pattern recognition receptors (PRRs). Implication of PRRs in vitiligo has been demonstrated in several GWAS, in particular genes encoding TLRs and their signaling pathway $(24,25)$. In addition, polymorphisms in NLRs have been described in patients with non-segmental vitiligo. Upregulated NLRP3 expression has been detected in perilesional keratinocytes in vitiligo skin and associated with higher cutaneous IL-1 $\beta$ expression and increased severity of the disease $(26,27)$.

Viral components are likely involved in vitiligo pathogenesis, as they can trigger activation of the immune system, however whether viruses can activate the innate immune response in the context of vitiligo is poorly described. Viruses possess several structurally diverse PAMPs, including surface glycoproteins, DNA, and RNA species (28). Virus infection could thus activate the innate response and potentially trigger a vitiligo flare. There is some evidence that viral infections in a genetically predisposed host may induce excessive ROS production by recruited lymphocytes leading to destruction of epidermal melanocytes (29). Furthermore, IFIH1, encoding intracellular virus sensor MDA5, has been identified as a vitiligo susceptibility gene capable of inducing secretion of CXCL10 and CXCL16 from keratinocytes and inducing infiltration of $\mathrm{CD}^{+} \mathrm{T}$ cells in vitiligo (30).

Bacteria are among the top producers of PAMPs and could directly trigger PRRs activation and therefore participate in activation of the innate immune response in vitiligo, however their direct role in triggering vitiligo has yet to be proven. While gut dysbiosis has been reported in several auto-immune disorders, there exists only one study suggesting skin dysbiosis in lesional zone of vitiligo patients compared to their non- 
lesional skin; however in that study there was no comparison to skin microbiota from healthy skin (31). The second study in mouse model of vitiligo treated with antibiotics has shown that depletion of certain bacterial strains in the gut induces skin depigmentation, suggesting possible gut-skin axis in the disease (32). We have recently demonstrated gut and skin dysbiosis in vitiligo compared to healthy controls; the most striking differences were seen in the deeper regions of vitiligo skin (33). Importantly, these changes were associated with mitochondrial damage and loss of protective bacteria at the same site with elevated systemic innate immunity in vitiligo patients.

\section{ROLE OF INNATE IMMUNE CELLS IN VITILIGO}

As suggested above, a large number of innate immunity genes that confer risk for vitiligo have been identified in genetic studies. Collectively, these papers undeniably support innate immunity pathways as critical in the development of the disease, which was further confirmed at the transcriptional level, with increased expression of innate immune related genes both in non-lesional and lesional skin of vitiligo patients $(10,11)$. If there is activation of innate immune pathways, naturally we would expect to see influx or activation of resident innate immune cells in the skin of vitiligo patients; however, the data in this area of research is only now starting to emerge. Although it has been known for a while that there is infiltration of macrophages, inflammatory DCs, dermal DCs, Langerhans cells, and NK cells to the leading edge or the lesional sites (34-37), their roles in vitiligo have not been thoroughly explored. The contribution of inflammatory DCs

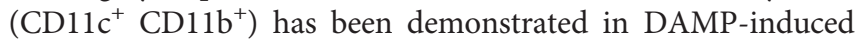
animal model of vitiligo driven by HSP70 (21), however their role in human disease remains to be proven. Studies dating over 10 years ago have demonstrated a positive correlation between levels of macrophage migration inhibitory factor (MIF) in the blood of vitiligo patients and their disease duration suggesting MIF may be a useful serum biomarker of vitiligo activity (38-40), however direct contribution of macrophages to the disease in the skin is unknown.

\section{Plasmacytoid Dendritic Cells}

Plasmacytoid dendritic cells (pDCs) certainly represent an important player in the initiation of the inflammatory response and the type I/II IFN signature in vitiligo skin. pDCs have been involved in various chronic inflammatory dermatoses, including cutaneous lupus erythematosus and psoriasis, mainly through their propensity to release high levels of IFN $\alpha$ (41). We showed that perilesional skin of vitiligo patients in the active phase of the disease harbors infiltrates of pDCs, associated with a local IFN response (36). Activation of this cell subset is likely mediated by the release of DAMPs from epidermal cells, as shown with HSP70i, potentiating IFN $\alpha$ secretion by pDCs and subsequent production CXCL9 and CXCL10 chemokines by epidermal cells, leading to the Th1 adaptive immune response establishment characteristic of vitiligo skin (42).
Furthermore, the other question which has been puzzling researchers was the initial source of the signature vitiligo cytokine IFN $\gamma$. We know that IFN $\gamma$ is critical for the progression of vitiligo through 1) its induction of CXCL9 and CXCL10 chemokines and thereby recruitment of $\mathrm{CD}^{+} \mathrm{T}$ cells expressing CXCR3, which are without doubt responsible for the loss of melanocytes and 2) its direct effect together with TNF $\alpha$ on melanocyte, through induction of melanocyte detachment from the basal layer of the epidermis (43). We recently highlighted that type 1 innate lymphoid cells (ILC1) are also poised to release $\operatorname{IFN} \gamma(37)$.

\section{NK Cells}

NK cells are described as a bridge between innate and adaptive immune system. They are characterized by their early and potent production of IFN $\gamma$. As discussed above, the transcriptional data supporting role of innate immunity in vitiligo is primarily based on differential gene expression associated with NK cell function, activity, and cytotoxicity $(10,11)$. It has been known for almost 30 years that there is an increase in circulating NK cells in the blood of vitiligo patients with abnormalities in their expression of inhibitory receptor CD158a and their activity (44-47), yet their role in vitiligo skin remained unexplored until recently. We have now confirmed the increased number of cytotoxic NK cells in not only the blood but also in the skin of vitiligo patients compared to healthy controls, predominantly in non-lesional skin (37). Furthermore, vitiligo NK cells are much more sensitive to stress, produce much larger amounts of IFN $\gamma$ following stress, and are directly implicated in initiation of long-term adaptive immunity against melanocytes (37).

\section{Innate Lymphoid Cells}

Innate lymphoid cells (ILCs) are the innate counterparts of $\mathrm{T}$ cells. In response to IL-12, IL-15, and IL-18, they secrete IFN $\gamma$; a signature vitiligo cytokine. We have recently demonstrated increased presence of ILC1 (but not ILC2 or ILC3) in vitiligo blood and skin and these cells to be the initial source of IFN $\gamma$, which is involved in early melanocyte apoptosis and subsequent T-cell mediated destruction of melanocytes (37).

\section{Melanocytes}

It has been known for a long time that melanocytes from vitiligo patients are intrinsically abnormal and are more sensitive to external stress $(48,49)$, however this defect alone doesn't explain the disease pathology as stressed melanocytes remain viable. As shown in a chicken model of spontaneous vitiligo, innate immunity is an important link between melanocyte stress and long-term adaptive immunity (50). Melanocytes have also been proposed as immunocompetent cells being able to process and present antigen, upregulate their own co-stimulatory markers and directly stimulate cytotoxic T lymphocytes following IFN $\gamma$ stimulation $(51,52)$. We have recently shown that human melanocytes express chemokine receptor of the B-isoform (CXCR3B), whose expression is upregulated in vitiligo melanocytes compared to healthy melanocytes and this receptor to play a critical role in anti-melanocyte immunity in vitiligo (37). 
Together, recent literature highlighted that innate immune pDCs, NK cells and ILC1 are capable of directly responding to stressed melanocytes and are critical in initiation of the disease, making these cells ideal primary target for therapeutic intervention.

\section{THERAPEUTIC PERSPECTIVES}

Vitiligo is a chronic inflammatory skin disorder and future therapeutic strategies might consider targeting the innate immunity side of the disease to halt initiation and/or progression of the disease, but such approach could also be envisioned as a maintenance therapy to prevent relapse.

Topical or systemic immunosuppressive drugs that are actually used for treating vitiligo such as corticosteroids, methotrexate or calcineurin inhibitors, have some potential impact on the innate immune response $(53,54)$. However, these agents have a broad impact on innate and adaptive immunity. Future approaches targeting more specifically the pathways involved in vitiligo could provide better responses with safer profile.

The elicitation of DAMPs depends on endoplasmic reticulum stress and oxidative stress. Many studies have described the role of the oxidative stress in vitiligo and how it can trigger the immune response (55-58). However, the efficacy of antioxidants in treating vitiligo is still a matter of debate as it relies on inconclusive studies or studies with contradictory results (59). This discrepancy between robust fundamental evidences and questionable clinical data could be explained by the differences in the type of antioxidant therapies used. More effective antioxidants with better bioavailability could effectively reduce the oxidative stress in the skin and provide a useful approach in treating vitiligo. There is increasing evidence showing mitochondrial alterations with increased production of ROS in vitiligo skin $(60,61)$. Compounds protecting specifically against this kind of mitochondrial damages could be of great interest in treating or preventing vitiligo relapses. Inhibition of DAMPs released by epidermal cells could also represent an interesting approach to prevent activation of innate cells. Indeed, mutant HSP70i have been shown to prevent auto-immune depigmentation or induce repigmentation both in mouse and Sinclair swine models $(21,22)$.

As detailed above, bacteria are major producers of PAMPs and alteration of skin and gut microbiome could participate in activation of the innate immune response in vitiligo (31-33). Modulating the skin or gut microbiome appears as an appealing approach. Recent data conducted in atopic dermatitis skin, demonstrated that topical formulation containing specific strains of probiotics could improve skin lesions (62). Additional studies are urgently needed, especially those in vitiligo, but modulation of microbiome, using prebiotics, probiotics, postbiotics, or fecal microbiota transplantation, could be an alternative approach for secondary prevention in vitiligo.

The development of antibodies targeting specifically the B isoform of CXCR3, could prevent the initial apoptosis of melanocytes and thus could be an effective preventive approach (37). Another strategy could rely on direct action on innate cells themselves. BDCA2 is a C-type lectin specifically expressed on pDCs, whose engagement inhibits the release of IFN $\alpha$. Of interest, the use of a monoclonal antibody targeting BDCA2 showed improvement of skin lesions in systemic lupus erythematosus (63), however no preclinical studies have been performed so far to evaluate the efficacy of such strategy in vitiligo. In line with the important role of IFN $\alpha$ in disease pathogenesis, hydroxychoroquine, a TLR7 and TLR9 inhibitor downregulating IFN $\alpha$ production by $\mathrm{pDCs}$, was shown to induce repigmentation of vitiligo lesions in a clinical case reports (64, $65)$. Whether inhibition of IFN $\alpha$ or its receptor could be an alternative strategy to block the type I IFN pathway in vitiligo has not yet been assessed.

NKG2D is one of the most frequent allelic variation found in vitiligo population. It also regulates both $\mathrm{NK}$ and $\mathrm{T}$ cell responses and thus, targets both innate and adaptive immune responses (66), and has been involved in vitiligo pathogenesis $(67,68)$. The use of anti-NKG2D antibodies could be a very promising approach for treating vitiligo.

In line with a therapy that would dampen both innate and adaptive immune response, IL-15 could represent another attractive strategy. Indeed, this cytokine is important for both $\mathrm{T}$ cells and NK cells maintenance and function. Recent studies highlighted the role of IL-15 on resident memory $\mathrm{T}$ cells in vitiligo pathogenesis and the interest to inhibit this cytokine or its receptor in vitiligo (68-70). Clinical phase II study is about to start, evaluating the efficacy of AMG714 for treatment of vitiligo (NCT04338581). Whether such targeting would also impact the innate response remains to be evaluated.

Targeting multiple cytokine pathways with JAK inhibitors is showing promising clinical outcome in vitiligo patients, as shown with the use of tofacitinib (blocking JAK1/3) or ruxolitinib (blocking JAK1/2) (71-73). Besides targeting IFN $\gamma$ signaling, such therapies will also likely block IFN $\alpha$ impact on epidermal cells, as this cytokine signals through JAK1/TYK2. Interestingly, a phase 2 clinical trial evaluating the efficacy of systemic administration of a JAK1/TYK2 inhibitor is ongoing (NCT03715829) and will provide new insights into the physiopathology of vitiligo.

\section{CONCLUSION}

Innate immunity has long time been overlooked in autoimmune disorders, including in vitiligo. However, from genetic and transcriptome data to modulation of key innate cells in vitiligo skin and blood, there are now accumulating and strong evidences supporting the key role of the innate immunity in pathogenesis of vitiligo (Figure 1). Activated by the PAMPs and DAMPs, the innate immunity appears as the bridge between potential triggering factors of vitiligo flares such as stress, Koebner phenomenon or infections, and the secondary activation of the adaptative immune response. These data foster new therapeutic 


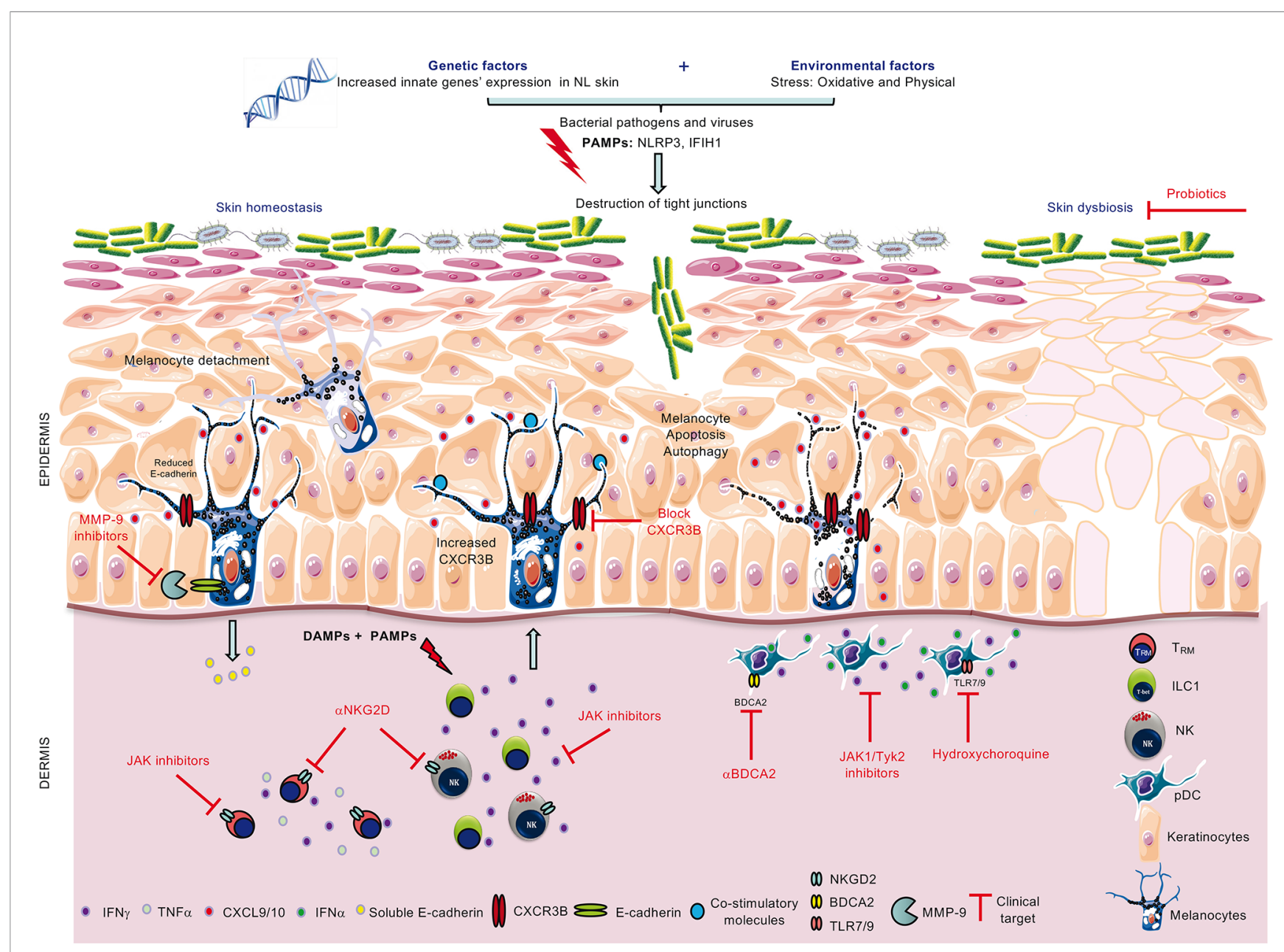

FIGURE 1 | Role of innate immunity in loss of melanocytes and therapeutic strategies to combat the disease. Combined genetic and environmental (PAMPs) or endogenous (DAMPs) stress activates innate immune cells ILC1/NK and pDCs to increase their production of IFN $\gamma$ and IFN $\alpha$, respectively. In turn, IFN $\gamma$ is involved in melanocyte detachment from the basal layer of the epidermis and upregulates melanocytic CXCR3B expression. In some melanocytes, CXCR3B activation induces melanocyte apoptosis which triggers increased expression of co-stimulatory molecules in other melanocytes and subsequent recruitment of cytotoxic $T$ cells. Possible clinical therapeutic targets include the use of anti-CXCR3B (to block initial apoptosis), JAK inhibitors (to inhibit cytokine release from ILC1, pDCs and memory $T$ cells, $T_{R M}$ ), anti-NKG2D (to block activating NKG2D receptor on NK and $T_{R M}$ ), anti-BDCA2 and hydroxychloroquine (to inhibit IFN $\alpha$ production from pDCs) and probiotics to restore skin homeostasis.

opportunities for vitiligo treatment but also for primary and secondary prevention. It will also be important to further characterize the role of the innate immune response in preventing repigmentation in patients with a stable disease.

\section{REFERENCES}

1. Boniface K, Seneschal J, Picardo M, Taïeb A. Vitiligo: Focus on Clinical Aspects, Immunopathogenesis, and Therapy. Clin Rev Allergy Immunol (2018) 54:52-67. doi: 10.1007/s12016-017-8622-7

2. Picardo M, Dell'Anna ML, Ezzedine K, Hamzavi I, Harris JE, Parsad D, et al. Vitiligo. Nat Rev Dis Primers (2015) 1:15011. doi: 10.1038/nrdp.2015.11

3. Willemsen M, Linkute R, Luiten RM, Matos TR. Skin-resident memory T cells as a potential new therapeutic target in vitiligo and melanoma. Pigment Cell Melanoma Res (2019) 32:612-22. doi: 10.1111/pcmr.12803

\section{AUTHOR CONTRIBUTIONS}

$\mathrm{KB}$, TP, JS, and MKT wrote the manuscript. All authors contributed to the article and approved the submitted version.

4. Riding RL, Harris JE. The Role of Memory CD8+ T Cells in Vitiligo. J Immunol (2019) 203:11-9. doi: 10.4049/jimmunol.1900027

5. Roberts GHL, Santorico SA, Spritz RA. The genetic architecture of vitiligo. Pigment Cell Melanoma Res (2020) 33:8-15. doi: 10.1111/pcmr.12848

6. Jin Y, Santorico SA, Spritz RA. Pediatric to Adult Shift in Vitiligo Onset Suggests Altered Environmental Triggering. J Invest Dermatol (2020) 140:241-243.e4. doi: 10.1016/j.jid.2019.06.131

7. Spritz RA, Santorico SA. The Genetic Basis of Vitiligo. J Invest Dermatol (2020) 41(2):265-73. doi: 10.1016/j.jid.2020.06.004

8. Jin Y, Andersen G, Yorgov D, Ferrara TM, Ben S, Brownson KM, et al. Genome-wide association studies of autoimmune vitiligo identify 23 new risk 
loci and highlight key pathways and regulatory variants. Nat Genet (2016) 48:1418-24. doi: 10.1038/ng.3680

9. Jin Y, Birlea SA, Fain PR, Ferrara TM, Ben S, Riccardi SL, et al. Genome-wide association analyses identify 13 new susceptibility loci for generalized vitiligo. Nat Genet (2012) 44:676-80. doi: 10.1038/ng.2272

10. Yu R, Broady R, Huang Y, Wang Y, Yu J, Gao M, et al. Transcriptome analysis reveals markers of aberrantly activated innate immunity in vitiligo lesional and non-lesional skin. PloS One (2012) 7:e51040. doi: 10.1371/ journal.pone.0051040

11. Regazzetti C, Joly F, Marty C, Rivier M, Mehul B, Reiniche P, et al. Transcriptional Analysis of Vitiligo Skin Reveals the Alteration of WNT Pathway: A Promising Target for Repigmenting Vitiligo Patients. J Invest Dermatol (2015) 135:3105-14. doi: 10.1038/jid.2015.335

12. Jadeja SD, Mayatra JM, Vaishnav J, Shukla N. Begum R. A Concise Review on the Role of Endoplasmic Reticulum Stress in the Development of Autoimmunity in Vitiligo Pathogenesis. Front Immunol (2020) 11:624566. doi: $10.3389 / \mathrm{fimmu} .2020 .624566$

13. Kim JY, Lee EJ, Seo J, Oh SH. Impact of high-mobility group box 1 on melanocytic survival and its involvement in the pathogenesis of vitiligo. $\mathrm{Br} \mathrm{J}$ Dermatol (2017) 176:1558-68. doi: 10.1111/bjd.15151

14. Becatti M. Oxidative stress and high-mobility group box 1 (HMGB1) protein release in vitiligo. Br J Dermatol (2017) 176:1436-7. doi: 10.1111/bjd.15538

15. Mou K, Liu W, Miao Y, Cao F, Li P. HMGB1 deficiency reduces H2 O2 -induced oxidative damage in human melanocytes via the Nrf2 pathway. J Cell Mol Med (2018) 22:6148-56. doi: 10.1111/jcmm.13895

16. Cui T, Zhang W, Li S, Chen X, Chang Y, Yi X, et al. Oxidative Stress-Induced HMGB1 Release from Melanocytes: A Paracrine Mechanism Underlying the Cutaneous Inflammation in Vitiligo. J Invest Dermatol (2019) 139:21742184.e4. doi: 10.1016/j.jid.2019.03.1148

17. Zhang Y, Liu L, Jin L, Yi X, Dang E, Yang Y, et al. Oxidative stress-induced calreticulin expression and translocation: new insights into the destruction of melanocytes. J Invest Dermatol (2014) 134:183-91. doi: 10.1038/jid.2013.268

18. Mosenson JA, Flood K, Klarquist J, Eby JM, Koshoffer A, Boissy RE, et al. Preferential secretion of inducible HSP70 by vitiligo melanocytes under stress. Pigment Cell Melanoma Res (2014) 27:209-20. doi: 10.1111/pcmr.12208

19. Mosenson JA, Eby JM, Hernandez C, Le Poole IC. A central role for inducible heat-shock protein 70 in autoimmune vitiligo. Exp Dermatol (2013) 22:566-9. doi: 10.1111/exd.12183

20. Denman CJ, McCracken J, Hariharan V, Klarquist J, Oyarbide-Valencia K, Guevara-Patiño JA, et al. HSP70i accelerates depigmentation in a mouse model of autoimmune vitiligo. J Invest Dermatol (2008) 128:2041-8. doi: $10.1038 /$ jid.2008.45

21. Mosenson JA, Zloza A, Nieland JD, Garrett-Mayer E, Eby JM, Huelsmann EJ, et al. Mutant HSP70 reverses autoimmune depigmentation in vitiligo. Sci Transl Med (2013) 5:174ra28. doi: 10.1126/scitranslmed.3005127

22. Henning SW, Fernandez MF, Mahon JP, Duff R, Azarafrooz F, Guevara-Patiño JA, et al. HSP70iQ435A-Encoding DNA Repigments Vitiligo Lesions in Sinclair Swine. J Invest Dermatol (2018) 138:2531-9. doi: 10.1016/j.jid.2018.06.186

23. Doss RW, El-Rifaie A-AA, Abdel-Wahab AM, Gohary YM, Rashed LA. Heat Shock Protein-70 Expression in Vitiligo and its Relation to the Disease Activity. Indian J Dermatol (2016) 61:408-12. doi: 10.4103/0019-5154.185704

24. Traks T, Keermann M, Karelson M, Rätsep R, Reimann E, Silm H, et al. Polymorphisms in Toll-like receptor genes are associated with vitiligo. Front Genet (2015) 6:278. doi: 10.3389/fgene.2015.00278

25. Karaca N, Ozturk G, Gerceker BT, Turkmen M, Berdeli A. TLR2 and TLR4 gene polymorphisms in Turkish vitiligo patients. J Eur Acad Dermatol Venereol (2013) 27:e85-90. doi: 10.1111/j.1468-3083.2012.04514.x

26. Li S, Kang P, Zhang W, Jian Z, Zhang Q, Yi X, et al. Activated NLR family pyrin domain containing 3 (NLRP3) inflammasome in keratinocytes promotes cutaneous T-cell response in patients with vitiligo. J Allergy Clin Immunol (2020) 145:632-45. doi: 10.1016/j.jaci.2019.10.036

27. Levandowski CB, Mailloux CM, Ferrara TM, Gowan K, Ben S, Jin Y, et al. NLRP1 haplotypes associated with vitiligo and autoimmunity increase interleukin-1 $\beta$ processing via the NLRP1 inflammasome. Proc Natl Acad Sci USA (2013) 110:2952-6. doi: 10.1073/pnas.1222808110

28. Mogensen TH, Paludan SR. Reading the viral signature by Toll-like receptors and other pattern recognition receptors. J Mol Med (Berl) (2005) 83:180-92. doi: 10.1007/s00109-004-0620-6
29. Dwivedi M, Laddha NC, Begum R. Viral causes of Vitiligo: A New Perspective for Vitiligo Pathogenesis. Virol Immunol J (2018) 2:000181. doi: 10.23880/VIJ16000181

30. Zhuang T, Yi X, Chen J, Kang P, Chen X, Chen J, et al. Intracellular virus sensor MDA5 exacerbates vitiligo by inducing the secretion of chemokines in keratinocytes under virus invasion. Cell Death Dis (2020) 11:453. doi: 10.1038/ s41419-020-2665-z

31. Ganju P, Nagpal S, Mohammed MH, Nishal Kumar P, Pandey R, Natarajan VT, et al. Microbial community profiling shows dysbiosis in the lesional skin of Vitiligo subjects. Sci Rep (2016) 6:18761. doi: 10.1038/srep18761

32. Dellacecca ER, Cosgrove C, Mukhatayev Z, Akhtar S, Engelhard VH, Rademaker AW, et al. Antibiotics Drive Microbial Imbalance and Vitiligo Development in Mice. J Invest Dermatol (2020) 140:676-687.e6. doi: 10.1016/ j.jid.2019.08.435

33. Bzioueche H, Simonyte Sjodin K, West C, Khemis A, Passeron T, Tulik MK. Analysis of Matched Skin and Gut Microbiome of Vitiligo Patients Reveals Deep Skin Dysbiosis: Link With Mitochondrial and Immune Changes. J Invest Dermatol (2021) S0022-202X(21)01006-X. doi: 10.1016/j.jid.2021.01.036

34. Wang CQF, Cruz-Inigo AE, Fuentes-Duculan J, Moussai D, Gulati N, Sullivan-Whalen $\mathrm{M}$, et al. Th17 cells and activated dendritic cells are increased in vitiligo lesions. PloS One (2011) 6:e18907. doi: 10.1371/ journal.pone. 0018907

35. Le Poole IC, van den Wijngaard RM, Westerhof W, Das PK. Presence of T cells and macrophages in inflammatory vitiligo skin parallels melanocyte disappearance. Am J Pathol (1996) 148:1219-28.

36. Bertolotti A, Boniface K, Vergier B, Mossalayi D, Taieb A, Ezzedine K, et al. Type I interferon signature in the initiation of the immune response in vitiligo. Pigment Cell Melanoma Res (2014) 27:398-407. doi: 10.1111/pcmr.12219

37. Tulic MK, Cavazza E, Cheli Y, Jacquel A, Luci C, Cardot-Leccia N, et al. Innate lymphocyte-induced CXCR3B-mediated melanocyte apoptosis is a potential initiator of T-cell autoreactivity in vitiligo. Nat Commun (2019) 10:2178. doi: 10.1038/s41467-019-09963-8

38. Serarslan G, Yönden Z, Sögüt S, Savaş N, Celik E, Arpaci A. Macrophage migration inhibitory factor in patients with vitiligo and relationship between duration and clinical type of disease. Clin Exp Dermatol (2010) 35:487-90. doi: $10.1111 / j .1365-2230.2009 .03617 . x$

39. Ma L, Xue H-B, Guan X-H, Shu C-M, Zhang Y-J, Zhang J-H, et al. Relationship of macrophage migration inhibitory factor levels in PBMCs, lesional skin and serum with disease severity and activity in vitiligo vulgaris. Braz J Med Biol Res (2013) 46:460-4. doi: 10.1590/S0100-879X2012007500152

40. Farag AGA, Hammam MA, Habib MS, Elnaidany NF, Kamh ME. Macrophage migration inhibitory factor as an incriminating agent in vitiligo. Bras Dermatol (2018) 93:191-6. doi: 10.1590/abd18064841.20186068

41. Conrad C, Gilliet M. Type I IFNs at the interface between cutaneous immunity and epidermal remodeling. J Invest Dermatol (2012) 132:175962. doi: $10.1038 /$ jid.2012.149

42. Jacquemin C, Rambert J, Guillet S, Thiolat D, Boukhedouni N, Doutre M-S, et al. Heat shock protein 70 potentiates interferon alpha production by plasmacytoid dendritic cells: relevance for cutaneous lupus and vitiligo pathogenesis. Br J Dermatol (2017) 177:1367-75. doi: 10.1111/bjd.15550

43. Boukhedouni N, Martins C, Darrigade A-S, Drullion C, Rambert J, Barrault C, et al. Type-1 cytokines regulate MMP-9 production and E-cadherin disruption to promote melanocyte loss in vitiligo. JCI Insight (2020) 5(11): e133772. doi: 10.1172/jci.insight. 133772

44. Halder RM, Walters CS, Johnson BA, Chakrabarti SG, Kenney JA. Aberrations in $\mathrm{T}$ lymphocytes and natural killer cells in vitiligo: a flow cytometric study. J Am Acad Dermatol (1986) 14:733-7. doi: 10.1016/ s0190-9622(86)70085-6

45. Basak PY, Adiloglu AK, Koc IG, Tas T, Akkaya VB. Evaluation of activatory and inhibitory natural killer cell receptors in non-segmental vitiligo: a flow cytometric study. J Eur Acad Dermatol Venereol (2008) 22:970-6. doi: 10.1111/j.1468-3083.2008.02681.x

46. Mozzanica N, Frigerio U, Negri M, Tadini G, Villa ML, Mantovani M, et al. Circadian rhythm of natural killer cell activity in vitiligo. J Am Acad Dermatol (1989) 20:591-6. doi: 10.1016/s0190-9622(89)70069-4

47. Mozzanica N, Villa ML, Foppa S, Vignati G, Cattaneo A, Diotti R, et al. Plasma alpha-melanocyte-stimulating hormone, beta-endorphin, met- 
enkephalin, and natural killer cell activity in vitiligo. J Am Acad Dermatol (1992) 26:693-700. doi: 10.1016/0190-9622(92)70094-v

48. Jimbow K, Chen H, Park JS, Thomas PD. Increased sensitivity of melanocytes to oxidative stress and abnormal expression of tyrosinase-related protein in vitiligo. Br J Dermatol (2001) 144:55-65. doi: 10.1046/j.1365-2133.2001.03952.x

49. Maresca V, Roccella M, Roccella F, Camera E, Del Porto G, Passi S, et al. Increased sensitivity to peroxidative agents as a possible pathogenic factor of melanocyte damage in vitiligo. J Invest Dermatol (1997) 109:310-3. doi: 10.1111/1523-1747.ep12335801

50. Shi F, Kong B-W, Song JJ, Lee JY, Dienglewicz RL, Erf GF. Understanding mechanisms of vitiligo development in Smyth line of chickens by transcriptomic microarray analysis of evolving autoimmune lesions. BMC Immunol (2012) 13:18. doi: 10.1186/1471-2172-13-18

51. Lu Y, Zhu W-Y, Tan C, Yu G-H, Gu J-X. Melanocytes are potential immunocompetent cells: evidence from recognition of immunological characteristics of cultured human melanocytes. Pigment Cell Res (2002) 15:454-60. doi: 10.1034/j.1600-0749.2002.02065.x

52. Le Poole IC, Mutis T, van den Wijngaard RM, Westerhof W, Ottenhoff T, de Vries RR, et al. A novel, antigen-presenting function of melanocytes and its possible relationship to hypopigmentary disorders. I Immunol (1993) 151:7284-92.

53. Passeron T. Medical and Maintenance Treatments for Vitiligo. Dermatol Clin (2017) 35:163-70. doi: 10.1016/j.det.2016.11.007

54. Chang H-C, Hsu Y-P, Huang Y-C. The effectiveness of topical calcineurin inhibitors compared with topical corticosteroids in the treatment of vitiligo: A systematic review and meta-analysis. J Am Acad Dermatol (2020) 82:243-5. doi: 10.1016/j.jaad.2019.07.108

55. Dell'Anna ML, Ottaviani M, Bellei B, Albanesi V, Cossarizza A, Rossi L, et al. Membrane lipid defects are responsible for the generation of reactive oxygen species in peripheral blood mononuclear cells from vitiligo patients. J Cell Physiol (2010) 223:187-93. doi: 10.1002/jcp.22027

56. Passeron T, Ortonne J-P. Activation of the unfolded protein response in vitiligo: the missing link? J Invest Dermatol (2012) 132:2502-4. doi: 10.1038/ jid.2012.328

57. Schallreuter KU, Salem MAEL, Holtz S, Panske A. Basic evidence for epidermal $\mathrm{H} 2 \mathrm{O} 2 / \mathrm{ONOO}(-)$-mediated oxidation/nitration in segmental vitiligo is supported by repigmentation of skin and eyelashes after reduction of epidermal $\mathrm{H} 2 \mathrm{O} 2$ with topical NB-UVB-activated pseudocatalase PC-KUS. FASEB J (2013) 27:3113-22. doi: 10.1096/fj.12-226779

58. Schallreuter KU, Moore J, Wood JM, Beazley WD, Gaze DC, Tobin DJ, et al. In vivo and in vitro evidence for hydrogen peroxide ( $\mathrm{H} 2 \mathrm{O} 2)$ accumulation in the epidermis of patients with vitiligo and its successful removal by a UVBactivated pseudocatalase. J Investig Dermatol Symp Proc (1999) 4:91-6. doi: 10.1038/sj.jidsp.5640189

59. Speeckaert R, Dugardin J, Lambert J, Lapeere H, Verhaeghe E, Speeckaert $\mathrm{MM}$, et al. Critical appraisal of the oxidative stress pathway in vitiligo: a systematic review and meta-analysis. J Eur Acad Dermatol Venereol (2018) 32:1089-98. doi: 10.1111/jdv.14792

60. Dell'Anna ML, Ottaviani M, Kovacs D, Mirabilii S, Brown DA, Cota C, et al. Energetic mitochondrial failing in vitiligo and possible rescue by cardiolipin. Sci Rep (2017) 7:13663. doi: 10.1038/s41598-017-13961-5

61. Sahoo A, Lee B, Boniface K, Seneschal J, Sahoo SK, Seki T, et al. MicroRNA211 Regulates Oxidative Phosphorylation and Energy Metabolism in Human Vitiligo. J Invest Dermatol (2017) 137:1965-74. doi: 10.1016/j.jid.2017.04.025

62. Myles IA, Castillo CR, Barbian KD, Kanakabandi K, Virtaneva K, Fitzmeyer E, et al. Therapeutic responses to Roseomonas mucosa in atopic dermatitis may involve lipid-mediated TNF-related epithelial repair. Sci Transl Med (2020) 12 (560):eaaz8631. doi: 10.1126/scitranslmed.aaz8631

63. Furie R, Werth VP, Merola JF, Stevenson L, Reynolds TL, Naik H, et al. Monoclonal antibody targeting BDCA2 ameliorates skin lesions in systemic lupus erythematosus. J Clin Invest (2019) 129:1359-71. doi: 10.1172/ JCI124466

64. Li D-G, Hu W-Z, Ma H-J, Liu W, Yang Q-Q, Zhao G. Hydroxychloroquine protects melanocytes from autoantibody-induced injury by reducing the binding of antigen-antibody complexes. Mol Med Rep (2016) 14:1275-82. doi: $10.3892 / \mathrm{mmr} .2016 .5354$

65. Joo K, Park W, Kwon SR, Lim MJ, Jung K-H. Improvement of vitiligo in a patient with rheumatoid arthritis after hydroxychloroquine treatment. Int $J$ Rheum Dis (2015) 18:679-80. doi: 10.1111/1756-185X.12442

66. Wensveen FM, Jelenčić V, Polić B. NKG2D: A Master Regulator of Immune Cell Responsiveness. Front Immunol (2018) 9:441. doi: 10.3389/ fimmu.2018.00441

67. Zloza A, Lyons GE, Chlewicki LK, Kohlhapp FJ, O'Sullivan JA, Lacek AT, et al. Engagement of NK receptor NKG2D, but not 2B4, results in self-reactive CD8+ T cells and autoimmune vitiligo. Autoimmunity (2011) 44:599-606. doi: 10.3109/08916934.2011.593599

68. Jacquemin C, Martins C, Lucchese F, Thiolat D, Taieb A, Seneschal J, et al. NKG2D Defines a Subset of Skin Effector Memory CD8 T Cells with Proinflammatory Functions in Vitiligo. J Invest Dermatol (2020) 140:11431153.e5. doi: 10.1016/j.jid.2019.11.013

69. Cheuk S, Schlums H, Gallais Sérézal I, Martini E, Chiang SC, Marquardt N, et al. CD49a Expression Defines Tissue-Resident CD8+ T Cells Poised for Cytotoxic Function in Human Skin. Immunity (2017) 46:287-300. doi: 10.1016/j.immuni.2017.01.009

70. Richmond JM, Strassner JP, Zapata L, Garg M, Riding RL, Refat MA, et al. Antibody blockade of IL-15 signaling has the potential to durably reverse vitiligo. Sci Transl Med (2018) 10(450):eaam7710. doi: 10.1126/ scitranslmed.aam7710

71. Phan K, Phan S, Shumack S, Gupta M. Repigmentation in vitiligo using janus kinase (JAK) inhibitors with phototherapy: systematic review and Metaanalysis. J Dermatolog Treat (2020) 1-5. doi: 10.1080/09546634.2020.1735615

72. Relke N, Gooderham M. The Use of Janus Kinase Inhibitors in Vitiligo: A Review of the Literature. J Cutan Med Surg (2019) 23:298-306. doi: 10.1177/ 1203475419833609

73. Rosmarin D, Pandya AG, Lebwohl M, Grimes P, Hamzavi I, Gottlieb AB, et al. Ruxolitinib cream for treatment of vitiligo: a randomised, controlled, phase 2 trial. Lancet (2020) 396:110-20. doi: 10.1016/S0140-6736(20)30609-7

Conflict of Interest: JS has been an advisor, speaker or investigator for Abbvie, Calypso Biotech, Lilly, Novartis, Pierre Fabre, Sanofi-Genzyme. KB has been an advisor, investigator for Calypso Biotech.

The remaining authors declare that the research was conducted in the absence of any commercial or financial relationships that could be construed as a potential conflict of interest.

Copyright (๑) 2021 Boniface, Passeron, Seneschal and Tulic. This is an open-access article distributed under the terms of the Creative Commons Attribution License (CC BY). The use, distribution or reproduction in other forums is permitted, provided the original author(s) and the copyright owner(s) are credited and that the original publication in this journal is cited, in accordance with accepted academic practice. No use, distribution or reproduction is permitted which does not comply with these terms. 